\title{
Experimental Method of Ultrasonic Sensor for Ions and Solutions Discrimination and Identification
}

\author{
Abdellah Mrij, Abdelmajid EL Bakkali, Jaouad Foshi
}

\begin{abstract}
The ultrasonic wave or vibration causes the deformation of the medium such as process of forming weld during construction of metallization contacts in semi-conductors. From that we propose to build a special circuit using ultrasonic sensor to discriminate mediums and materials. The spread of wave made by multipath limits the transmission speed. Generally, if the delay spread is the bigger so velocity is low. Thereon, we study the influence of ions in various solutions on the pressure by result on the directivity of ultrasonic wave. Each chemical solution has its appropriate properties that affect ultrasonic wave pressure according to the compression and dilatation of the medium. The goal of the current work is determination of ultrasonic velocity by result identification of chemical solutions. Above that the used method permit to propose an approach that permit to predict the ultrasonic wave velocity and the matching impedance of a mixture of mediums referring to their chemical composition.
\end{abstract}

Keywords: Ultrasonic Wave, Materials, Impedance, Ultra-sonic velocity.

\section{INTRODUCTION}

The ultrasonic waves are out hearing domain such as electromagnetic radiations and electromagnetic fields that both refer to the entire range of the electromagnetic spectrum, from extremely low frequencies to radio waves $[1,2]$. In practice, the second type is widely used because radiation sounds scary and its use may create danger in X-ray machines and radioactive material. Examples of electromagnetic radiation to increase frequency, are extremely low frequency (ELF), very low frequency (VLF), radio waves, microwaves, infrared (heat), visible light, ultraviolet, Xrays, and gamma rays [3]. All electromagnetic radiations travel at the speed of light. Metamaterials are characterized by its super absorption. These materials are made of meta-molecules. The structural design of a metamaterial presents exotic properties such as negative refraction [4-7], invisibility cloaks [8-11], subwavelength focusing [12, 13], or super absorption [14 -19]. Many methods are being developed to inspect the nature of materials but the present work has appropriate technique to

\section{Revised Manuscript Received on December 15, 2019}

Abdellah Mrij, Team Electronics, Instrumentation and Measurement, Department of Physics, Faculty of Sciences and Techniques, University Moulay Ismail, BP 509, Boutalamine, Errachidia, Morocco.

Abdelmajid EL Bakkali, Team Electronics, Instrumentation and Measurement, Department of Physics, Faculty of Sciences and Techniques, University Moulay Ismail, BP 509, Boutalamine, Errachidia, Morocco.

Jaouad Foshi, Team Electronics, Instrumentation and Measurement, Department of Physics, Faculty of Sciences and Techniques, University Moulay Ismail, BP 509, Boutalamine, Errachidia, Morocco. discriminate solutions content and make adequate characterization. The aim is an exploiting of ultrasonic wave transmission velocity to identify the nature of a given ions therein a solution. To resume the goal is to adopt this method under resonance frequency around $40 \mathrm{KHz}$ like calibration for typical operational power levels used in abundance estimation to identify the mixture of ions [20-24].Moreover, the coefficients of composition are very significant to expect the content and deduct the impedance, velocity and compressibility factor.

\section{EXPERIMENTAL SETUP}

The adopted system of ultrasonic waves is using a simple emitter and receiver as suitable transducer provided with an amplification circuit to get appropriate echoed voltage to be analyzed. The principle is given by the schematic that can be depicted by the following figure 1 .

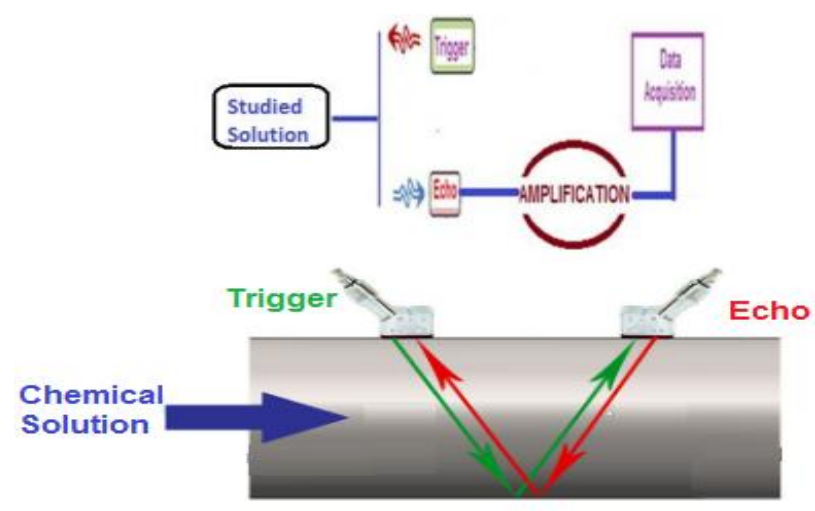

Fig. 1. Principle of emission and reception of ultrasonic waves.

Non-destructive testing involves the use of ultrasonic echo location to gather information on the integrity of the mechanical structures. Since changes in the material present impedance mismatch from which an ultrasonic wave is reflected, ultrasonic testing can be used to identify faults, holes and corrosion in materials in the way to inspect the nature of the element. An acoustic emission has resolved many field problems as it is efficient to identify the kind of material before integrate it in industrial and smart technologies. The ultrasonic delay is different by dependence on each metal or mineral property. Thereon we study here the effect of different ions in aqueous solutions.

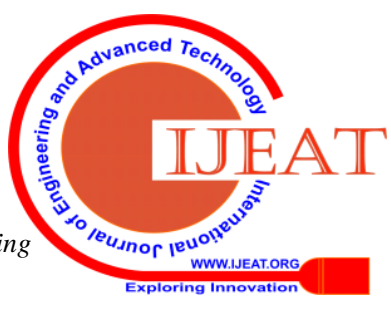


The simplified schematic built in autotrax software is given in the Figure 2.

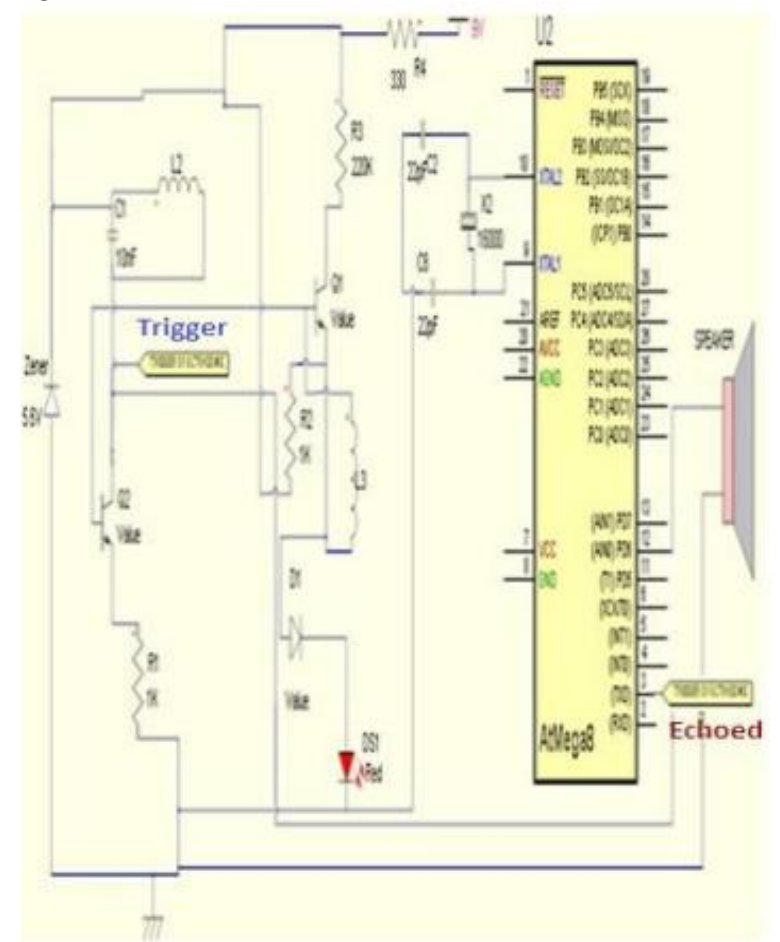

Fig. 2. Detector circuit for control of emitted and echoed waves.

The principle of solutions and ions discrimination is based on changing of compression and dilatation according to temperature variation as we can illustrate by the following synoptic schematic.

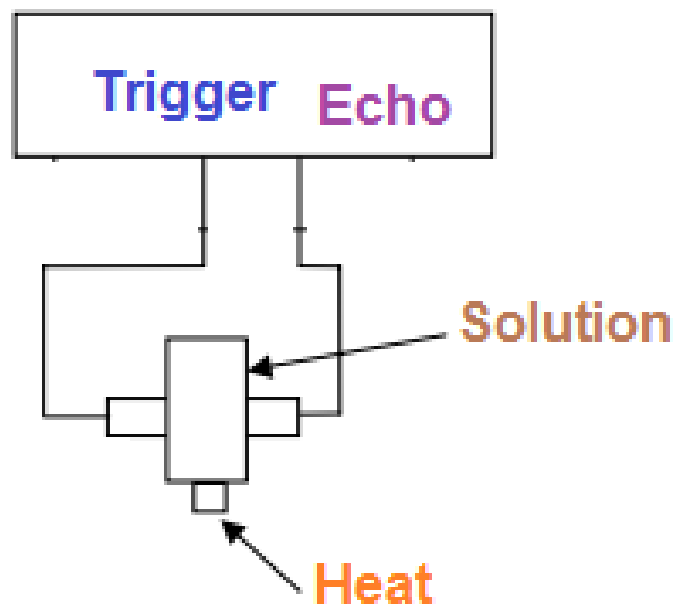

Fig. 3. Synoptic schematic of velocity measurement.

The fabricated realization is shown by the following image as given in the Figure 3. The existence of a given ions make different impact on the emitted and reflected wave. Indeed, the density varies and leads into a special absorption and variation of the echoed wave. Hence each ion has a specific ratio due its effect on compression. Besides, to prevent any losses we added amplification circuit to boost transmission and reflection. We exploit these concepts to discriminate ions by measurement of reflected voltage amplitude, attenuation coefficient, reflectance and impedance.

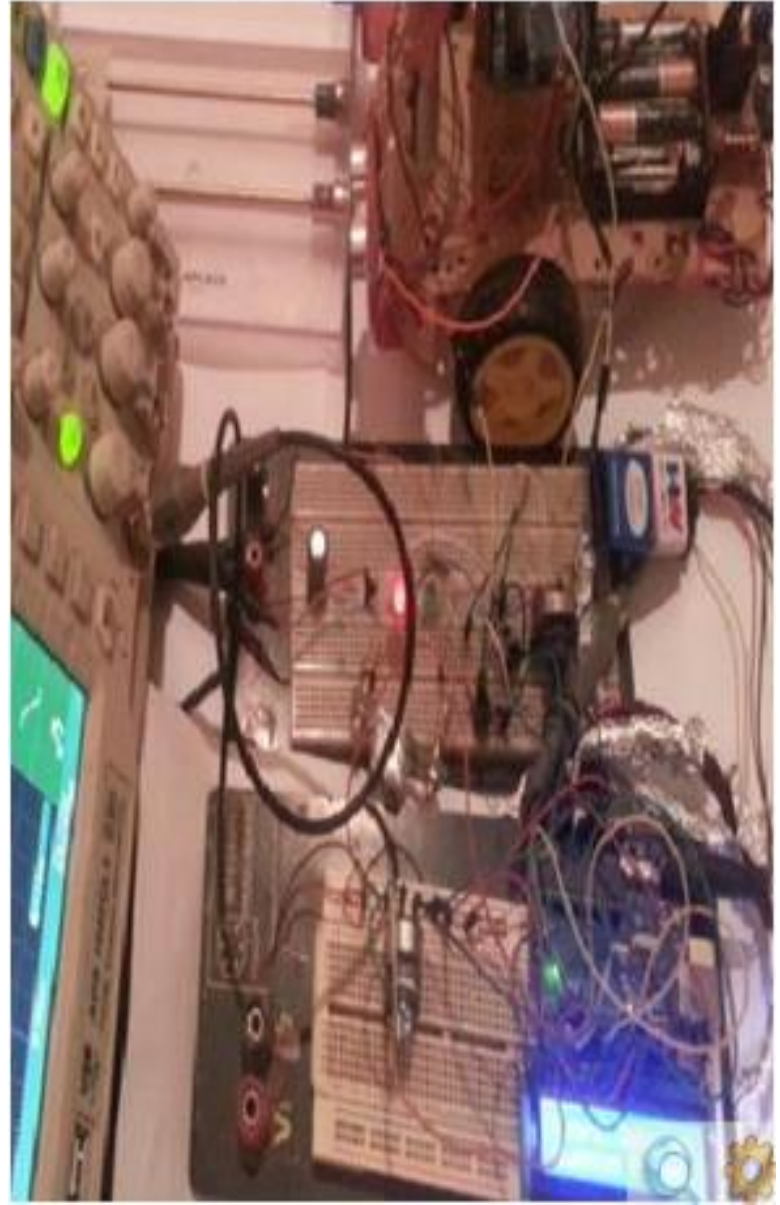

Fig. 4. Experimental system circuit.

\section{RESULTS AND DISCUSSIONS}

Some ions are privileged among others talking about ultrasonic wave propagation performances. Indeed, each type is characterized by its appropriate transmission so the directivity gain and the power radiates are varying depending on the pressure of the ultrasonic wave.. Moreover, many parameters are challenging such as the frequency, the concentration and the temperature of solution. Above that, the number of kind in aqueous medium is very significant for ultrasonic wave transmission due impact on compression and dilatation of medium phases. The justification of an accurate propagation is confirmed by measurement of velocity and minimization of delay to receive echoed wave. We give below a simulation of ultrasonic wave where the directivity depends on the pressure. We notice that the pressure has Pascal unity $(\mathrm{Pa})$ however the angle is on radian ( $\mathrm{Rad})$. The elapsed time by ultrasonic wave as evoked is varying with concentration, temperature and the frequency of the wave. Thereafter, we keep the distance between ultrasonic emitter and the specimen as border equal to $20 \mathrm{~cm}$. The simulation of directivity and pressure are given in the Figure 4 and Figure 5. 


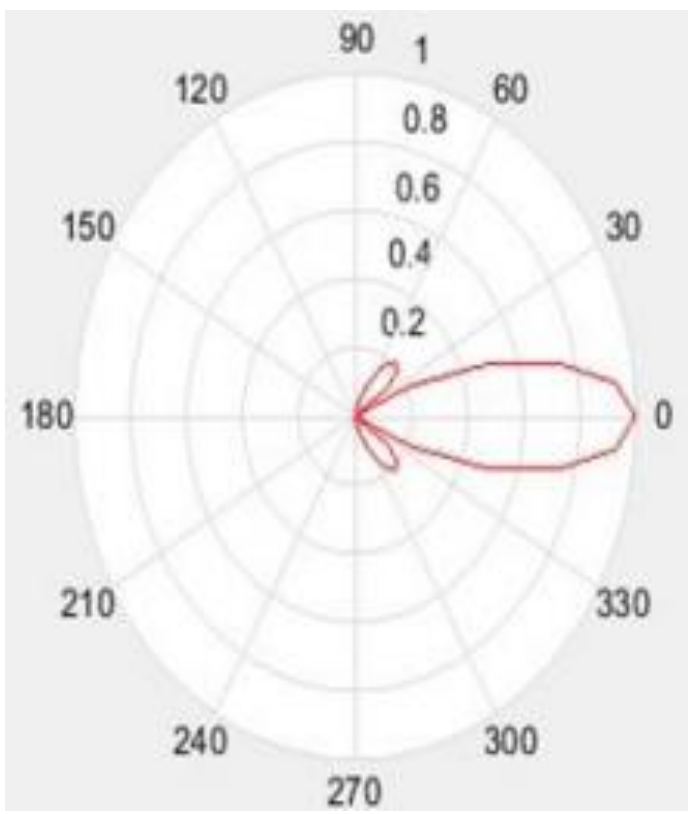

Fig. 5. Ultrasonic wave directivity with various pressures.

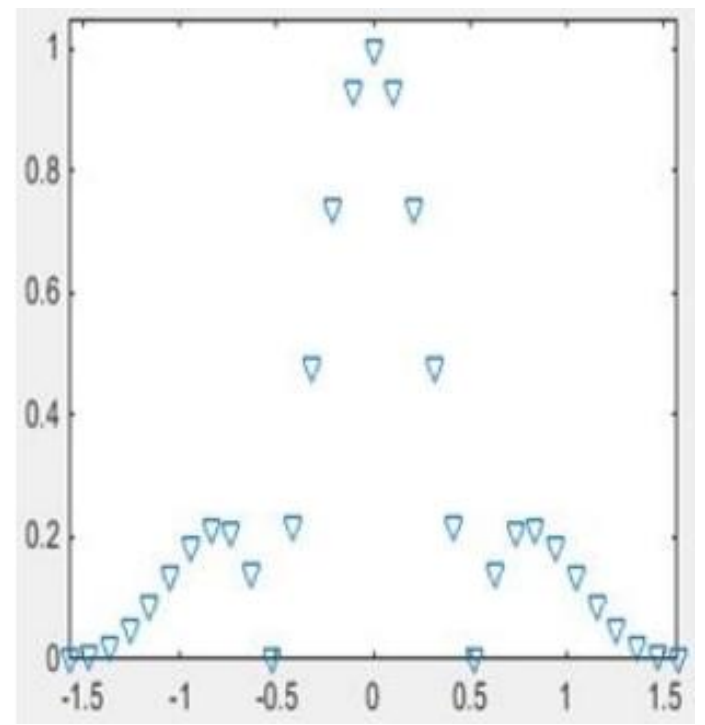

Fig. 6. Ultrasonic vibration pressure with angle variation.

The reflected intensity from the material is received by an accurate circuit that contains amplification to protect the signal from losses and direct it into Arduino board and digital oscilloscope to be analyzed and get required parameters. Thus, each solution has special impedance so we determine it with velocity by ultrasonic sensor to characterize ions. In fact, the suitable values are obtained at resonance frequency. Another concept is that due the difference of impedance the attenuation of amplitude is observed. For example the attenuation coefficient of water transmission medium is $(0.0022 \mathrm{~dB} / \mathrm{cm} . \mathrm{MHz})$. Above, the angle of emission affects the directivity of the ultrasonic waves. Moreover, the tension of the medium $\mathrm{T}$ has influence on the velocity using longitudinal mass $\mu$. It seems that ions have influence on the directivity by result we make comparisons of average and accumulated measurements such as reflected voltage, velocity, impedance and attenuation for different ions as we recapitulate in the following table.
Table 1: Measurements for some mediums.

\begin{tabular}{|l|l|l|l|l|l|l|}
\hline Material & $\begin{array}{l}\text { Reflected } \\
\text { Voltage }\end{array}$ & \multicolumn{2}{l|}{ Density } & Velocity & Impedance & Attenuation \\
\hline $\mathrm{Cl}-$ & 2.7 & 3.2 & 206 & 0.65 & -6 \\
\hline $\mathrm{Al} 3+$ & 1.6 & 2.7 & 5100 & 13 & -3 \\
\hline $\mathrm{Zn} 2+$ & 3 & 7.1 & 3700 & 26.3 & -4 \\
\hline $\mathrm{Ag}+$ & 2.5 & 10.5 & 26 & 27.4 & -6 \\
\hline $\mathrm{Cu} 2+$ & 1.5 & 8.9 & 3570 & 31.9 & -5 \\
\hline
\end{tabular}

Measurements of ultrasound velocity for some solutions at

$20 \mathrm{C}^{\circ}$ :

\begin{tabular}{|l|l|}
\hline Solution & Velocity $(\mathrm{m} / \mathrm{s})$ \\
\hline FeSO4 & 1650 \\
\hline Distilled Water & 1507 \\
\hline Methanol & 1200 \\
\hline Ethanol & 1281 \\
\hline
\end{tabular}

Measurements of ultrasound velocity for some solutions at $25 \mathrm{C}^{\circ}$ :

\begin{tabular}{|l|l|}
\hline Solution & Velocity $(\mathrm{m} / \mathrm{s})$ \\
\hline FeSO4 & 1658 \\
\hline Distilled Water & 1513 \\
\hline Methanol & 1209 \\
\hline Ethanol & 1292 \\
\hline
\end{tabular}

Measurements of ultrasound velocity for some solutions at $30 \mathrm{C}^{\circ}:$

\begin{tabular}{|l|l|}
\hline Solution & Velocity $(\mathrm{m} / \mathrm{s})$ \\
\hline FeSO4 & 1661 \\
\hline Distilled Water & 1518 \\
\hline Methanol & 1214 \\
\hline Ethanol & 1301 \\
\hline
\end{tabular}

Measurements of ultrasound velocity for some solutions at $35 \mathrm{C}^{\circ}:$

\begin{tabular}{|l|l|}
\hline Solution & Velocity $(\mathrm{m} / \mathrm{s})$ \\
\hline FeSO4 & 1670 \\
\hline Distilled Water & 1523 \\
\hline Methanol & 1219 \\
\hline Ethanol & 1307 \\
\hline
\end{tabular}

The following curve represents the variations of velocity for different ions. The evolution is depending on their intrinsic properties such as viscosity and density as we can clearly see in Figure 7. 


\section{Experimental Method of Ultrasonic Sensor for Ions and Solutions Discrimination and Identification}

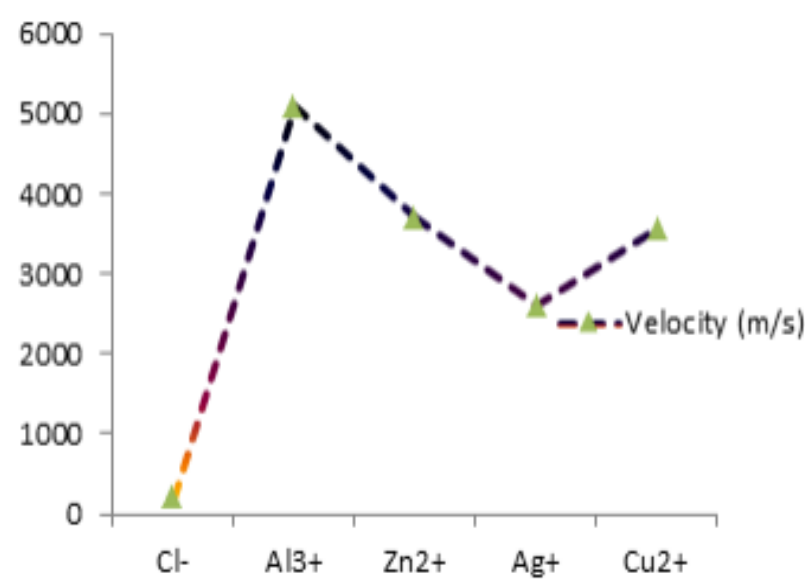

Fig. 7. Transmission velocity of ultrasound in many solutions.

The ions have different properties that are significant for ultrasonic propagation. The ultrasonic sensor works under adequate power to perform its operation. Above, the study in this work focuses on aqueous mediums so the mechanical properties are varying with various parameters such as temperature, concentration, viscosity and nanostructure. Thereon, we restrict the measurements here on specific evolutions of velocity, impedance and attenuation. By the way we establish the prediction of content while we study an hybrid medium of ions. After calculation of attenuation we obtain the reflected voltage of studied elements as depicted in the Figure 8.

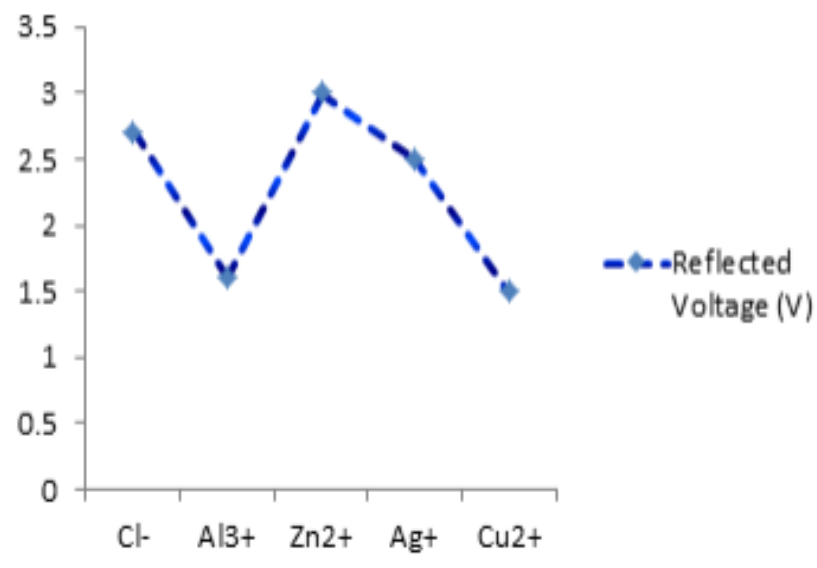

Fig. 8. Reflected voltage evolution by solutions

The reflected power of ultrasonic wave is a crucial characteristic. Indeed, It describes how a material transmits and reflects the wave's energy like transmittance. Both reflectance and transmittance are describing the manner in which the energy is spreading. We confirm the performances when the prediction is able to identify the elements and their compositions. Otherwise that many perturbations are encountered during experiment process however we try to minimize the losses as possible. The impedance is changing with the curve below.

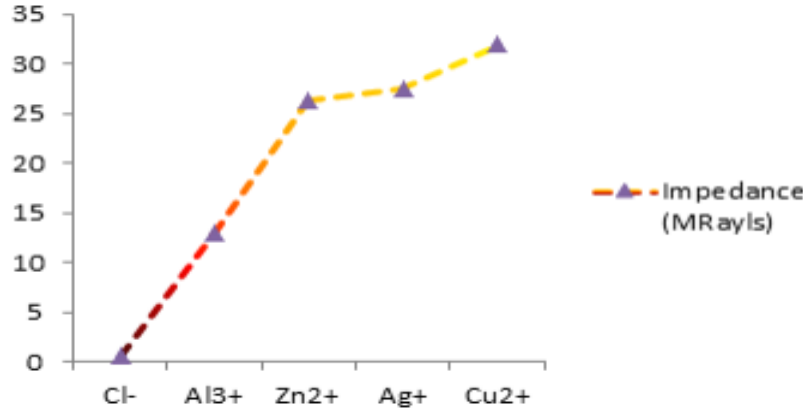

Fig. 9. Impedances of various mediums.

As noted previously each material has its effective impedance. Thereon we illustrate the impedance of some elements separately and some mixture as hybrid mediums. Referring to this concept we look for precision by getting convergence of impedance, velocity and attenuation into theorist values. The attenuation is given in the Figure 10 .

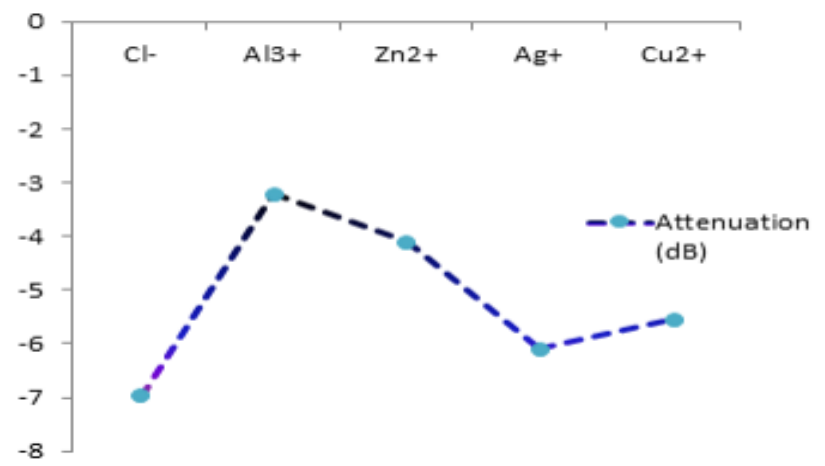

Fig. 10. Attenuation of power for specimens.

To identify and discriminate elements the calculated parameters are then compared to theoretical values. The result is an error found from the difference. The suitable measurement is the one with an error that has a tendency to zero. The study is fruitful while we are near to the real value that discriminates the type of ions. From these results the integration of ultra-sonic waves in materials characterization is very significant to determine matching parameters. The transmittance increases with directivity. Moreover, the echoed wave is depending on the reflectance of studied ions. According to the literature the resonance frequency influences on the determination of velocity, attenuation and impedance of aqueous solutions. Moreover, when we study a multi composition as expected and predicted by Desilets and Fraser model. Referring to this model, we can expect the values of impedances for each layer that depends on the effective impedance $\mathrm{Zf}$ at the free resonance frequency and the characteristic of the acoustic impedance of the front layer $\mathrm{Za}$. In parallel to mixture of solid layers we adopt the same principle to aqueous solution of many ions, the impedance of ions layer is based on the impedance of the previous one. 
At the case of three layers or three kinds of ions as an heterogeneous solution we can describe each layer's impedance by the expression given in reference of Desilets and Fraser model. We conclude that fluctuations affect negatively the precision of parameters calculation .As a result the matching-layer impedance justifies the effectiveness of the present method to improve Desilets and Fraser model accuracy during prediction of layer impedance. To reduce the difference between effective and matching layer impedance the quality of sensor as transducer is very significant. Each mineral has its appropriate reflectance $\mathrm{r}$ and impedance $\mathrm{Z}$. Thereon, by adjusting the distance and emission resonance frequency we expect the nature of medium. Therefore, its identity is defined. The directivity of ultrasound permits to minimize errors during emission and reflection intensity measurement. Consequently, we converge to real values of impedances, velocities and reflectance's.

\section{CONCLUSION}

The ultrasonic waves provided by suitable transducer are characterized by their suitable directivity and the pressure to be reflected with accepted sensibility. They also permit an accurate analysis of the echoed signal. The demonstrated transducer optimization ensures acoustic matching layer impedance with accepted precision. This method is fruitful to discriminate mediums and expect the nature which it depends on many parameters such as temperature, viscosity. The recent technique has many advantages especially a scanning of aqueous solutions using ultrasonic sensors for wide range. The calibration of sensors ensures good performances. To resolve extra setup for new technology that requires smart materials the properties of substances should be analyzed before implantation processes. Thereon, the ultrasonic wave is an alternative method to detect and identify nature and determine the physics and chemistry properties and also make adequate integration. Geology is an unlimited treasure and exploiting it in a good way offers a multitude of usages to make our life much better and easier.

\section{REFERENCES}

1. Smith, F.: EMF Protection AND Energy Enhancement, EMF REMEDI-ATION, Advance Clinical Nutrition. pp1-30 (2006).

2. Hajdarevic, K.; Pattinson, C.; Hadzic, A.; Hadzigrahic , J.: New Forensic Method for Measuring Dynamic Changes of EMF Level Limits Using Smart Dust, Information, Communication and Automation Technologies (ICAT). pp 1-5(2011).

3. Zamanian, A.; Hardiman, C.: Electromagnetic Radiation and Human Health: A Review of Sources and Effects, High Frequency Electronics, Summit Technical Media. pp 16-26 (2005).

4. Pendry, J.: Phys. Rev. Lett. 85, 3966 (2000).

5. Shelby, R.; Smith, R.; Schultz, S.: Science 292, 77 (2001).

6. Valentine, J.; Zhang, S.; Zentgraf, T.; Ulin-Avila, E.; Genov,A.; Bartal, G. : Xiang Zhang, Nature 455, 376 (2008).

7. S. Zhang, L. ;Yin, N. : Phys. Rev. Lett.99, 194301 (2009).

8. Schurig, D.; Mock, J.; Justice, B.; Cummer, S.; Pendry, Starr, F.; Smith,D. : Science 314, 977 (2006).

9. Chen, H.; Chan, C.: Appl. Phys. Lett. 91, 183518 (2007).

10. Torrent, D; Sanchez Dehesa, J.: New J. Phys. 10, 023004 (2008).
11. Cummer, S; Popa, I.; Schurig, D.; Smith, R.; Pendry, J.;Rahm, M.; Starr,A.: Phys. Rev. Lett. 100, 024301 (2008).

12. Lerosey, G.; Rosny, J.; Tourin, A.; Fink, M. : Science 315, 1120 (2007)

13. Lemoult, F.;Fink, M.; Lerosey, G.: Phys. Rev. Lett. 107064301 (2011).

14. Landy, N.; Sajuyigbe, S.; Mock, J.; Smith, D.; Padilla, W. :Phys. Rev.Lett. 100, 207402 (2008).

15. Ivansson, S.; Acoust, J.: Soc. Am. 124, 1974 (2008).

16. Moreau, A.; Ciraci, C.; Mock, J.; Hill, R.; Wang, Q.; Wiley, B.; Chilkoti, A.; Smith, D. : Nature 492, 86-89 (2012).

17. Mei, J.;Ma, G.; Yang, M; Wen, W;Sheng, P.: Nature Communications 3, 756 (2012).

18. Ma, G.; Yang, M.; Xiao, S.; Yang, Z.;Sheng, P: Nature Materials 13, 873 (2014).

19. Mortensen, N.; Willatzen, M.;Sanchez-Morcillo, V.:Scientific Reports, 4, 4674 (2014).

20. Tichy, F.; Solli, H.; Klaveness, H.:'Nonlinear effects in a $200-\mathrm{kHz}$ sound beam and consequences for target strength measurement", ICES Journal of Marine Science, 60, 571-574 (2003).

21. Norway, H. : Originally published as Simrad News Bulletin, Simrad AS (now Kongsberg Maritime AS), March, 2 p (2002).

22. Baker, A.; Lunde, P.: "Nonlinear propagation from circular echo-sounder transducers. Numerical simulation results", CMR Technical Note CMR-TN01-A10010-rev-01, Christian Michelsen Research AS, Bergen, Nor-way(August 2011), 23 p. De-classified revision of CMR Technical Note CMR-TN01-F10010 , , (confidential), February, 22 p (2001).

23. Baker, A.; Lunde, P.: "Nonlinear effects in sound propagation from echo-sounders used in fish abundance estimation. Numerical simulation results", CMR Technical Note CMR-TN02-A10008-rev-01, Christia

Michelsen Research AS, Bergen, Norway (August 2011), 27 p. De-classified revision of CMR Technical Note CMR-TN02-F10008 ,(confi-dential), April 26 p ( 2002).

24. Pedersen, A.; Lunde, P.; Vestrheim, M.: "Nonlinear sound propagation effects in fisheries research echosounders,-Measurements and simula-tions in freshwater", in Proc. of 28th Scandinavian Symposium on

Physical AcousticsUstaoset, Norway, 23-26 January 2005 Kristiansen, U. R. (ed.), The Norwegian Physical Society (CDROM only, ISBN 82-8123-000-2). , June (2005).

25. DeSilets, C. S. ; Fraser, J. D. ;Kino, G. S. : IEEE Trans.Son. Ultrason.SU-25, 115 (1978).

26. Goll, J. H. : IEEE Trans. Son. Ultrason. SU-26, 385 (1979).

27. Pozar, D. M.: Microwave Engineering (Wiley, New York), Chap. 5 (1988). 